\title{
Toward an evidence-based approach to whiplash injuries
}

\author{
Robert W Teasell MD FRCPC ${ }^{1,2}$, J Andrew McClure BA ${ }^{1}$, David Walton PhD candidate ${ }^{3}$
}

$\mathrm{W}$ hiplash-associated disorder (WAD) represents a major public health problem in Canada and is associated with significant socioeconomic costs throughout the industrial world. There are few entities that are both as common and as controversial as whiplash injuries. Moreover, whiplash injuries are more often managed based on opinion than actual evidence, a situation that has often been exploited by the medicolegal system with the encouragement of extreme viewpoints. Yet, despite the substantial costs, impact and uncertainty regarding the treatment of whiplash injuries, little funding has been devoted to research.

To determine the effectiveness of various treatments for whiplash-associated injuries, we conducted a systematic review similar to successful reviews we have conducted in stroke rehabilitation (1), acquired brain injury (2) and spinal cord injury (3). We were able to identify 83 studies that evaluated an intervention for whiplash injuries, 40 of which were randomized controlled trials. For a condition as common as WAD, there remains a paucity of research. However, from the evidence base described above, we were able to formulate several conclusions. For the purpose of providing a more relevant review of the evidence, we divided the interventions along temporal lines with three categories: acute care (interventions initiated within the first two weeks after injury), subacute care (interventions initiated from two to 12 weeks after injury) and chronic care (interventions initiated more than 12 weeks after injury). As a consequence of the natural history of WAD, treatments that have an impact in the acute phase will likely not have the same impact in the chronic phase; appreciating this is important, particularly in the absence of randomized controlled trials.

The strongest evidence was for the treatment of acute WAD. Perhaps not surprisingly, there is strong evidence that both physiotherapy and simple mobilization exercises result in greater improvement in cervical pain than rest and immobilization of the neck. Further research is needed to differentiate which activity/mobilization approaches are the most effective treatments. There is also evidence to suggest that simply advising a patient to 'act as usual' may be as effective as active therapy. In contrast, it does not appear that educational interventions offer significant benefits. While it has long been espoused that simply reassuring patients that they are likely to recover is in and of itself an important part of management, research suggests it has little, if any, impact on the outcome; the sole exception to this appears to be advice to stay active.
Thus, while it is important to avoid immobilization during the acute phase, the specific intervention used may not matter, whether it be formal physiotherapy, mobilization exercises or simply advice to remain active.

The evidence base was least developed for subacute interventions and, consequently, definitive statements regarding the effectiveness of interventions during this phase could not be made. Interestingly, the literature does not provide substantial support for the use of activation-based therapies during the subacute stage, although this may be more reflective of the limited research base than an actual lack of effectiveness. However, there is some evidence that progressive strengthening exercises may actually have an adverse long-term effect on pain and functional recovery when compared with advice to remain active. This suggests that although exercise and staying active may be beneficial early on, aggressive treatment approaches such as progressive strengthening exercises are not beneficial. A re-evaluation of how aggressive therapy sessions should be is needed. Moreover, this implies that work-hardening programs and functional restoration programs, which tend to have a significant strengthening component, are best avoided for the first three months.

Similar to the conclusions regarding interventions in acute WAD, exercise programs appear to be the most strongly supported intervention for patients with chronic disorders, although many questions remain regarding the relative effectiveness of various exercise protocols. For patients with chronic symptoms who do not respond to conventional treatments, it appears that radiofrequency neurotomy may be the most effective treatment option. The success of radiofrequency neurotomy in a significant number of patients with chronic WAD suggests that cervical facet joint injuries are a common source of pain. Nevertheless, this treatment has been incorporated slowly and with some reluctance, perhaps because pain relief is not permanent. By contrast, the use of botulinum toxin injections is becoming increasingly popular, despite the fact that there is conflicting evidence regarding its effectiveness. Botulinum toxin injections represent a promising but, as yet, unproven intervention in the treatment of WAD.

As we will carefully review in the following five articles, an evidence base for interventions for whiplash injuries is emerging and confirms what we have known for some time: immobilization is detrimental, while activity is beneficial. However, it also suggests that just because some exercise is

${ }^{1}$ Lawson Health Research Institute, Department of Physical Medicine and Rehabilitation, Parkwood Hospital; ${ }^{2}$ Schulich School of Medicine and Dentistry; ${ }^{3}$ School of Physical Therapy, Faculty of Health Sciences, University of Western Ontario, London, Ontario

Correspondence: Dr Robert W Teasell, Department of Physical Medicine and Rehabilitation, Parkwood Hospital, St Joseph's Health Care,

801 Commissioners Road East, London, Ontario N6C 5J1. Telephone 519-685-4000 ext 44559, fax 519-685-4023,

e-mail robert.teasell@sjhc.london.on.ca 
good, more is not necessarily better, and progressive strength training may not produce as good an outcome as simple advice to remain active. Reassurance of a good outcome does not appear to have any measurable benefit and, of course, for some individuals it is not accurate. The time has come to re-evaluate the 'harm versus hurt' concept, which suggests that recovery is not necessarily impeded by the experience of pain. This concept appears to be too simplistic for whiplash injuries in general. A better approach may be to acknowledge that some pain is inevitable with activity but that too much pain should be avoided. The fact remains that whiplash injuries recover much like other serious musculoskeletal injuries - most get better but a significant number do not; initial severity predicts recovery; exercise helps but too much is detrimental; and psychological factors are poor predictors of recovery but may affect coping.
The time has come to begin to promote an evidence-based approach to whiplash injuries. Further research is needed to determine which treatments are most effective at reducing the disabling symptoms associated with WAD and should be targeted to those areas where research is lacking.

\section{REFERENCES}

1. Teasell RW, Foley NC, Salter K, Bhogal SK, Jutai J, Speechley MR. Evidence-based review of stroke rehabilitation, 13th edn. $<$ www.ebrsr.com> (Accessed on October 8, 2010).

2. Teasell RW, Marshall S, Cullen N, et al. The evidence-based review of moderate to severe acquired brain injury, 6th edn. $<$ www.abiebr.com> (Accessed on October 8, 2010).

3. Eng JJ, Teasell RW, Miller WC, et al. Spinal cord injury rehabilitation evidence, $3 \mathrm{rd}$ edn. <www.scireproject.com> (Accessed on September 9, 2010). 


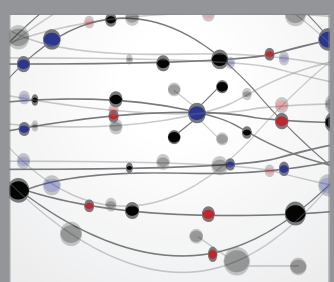

The Scientific World Journal
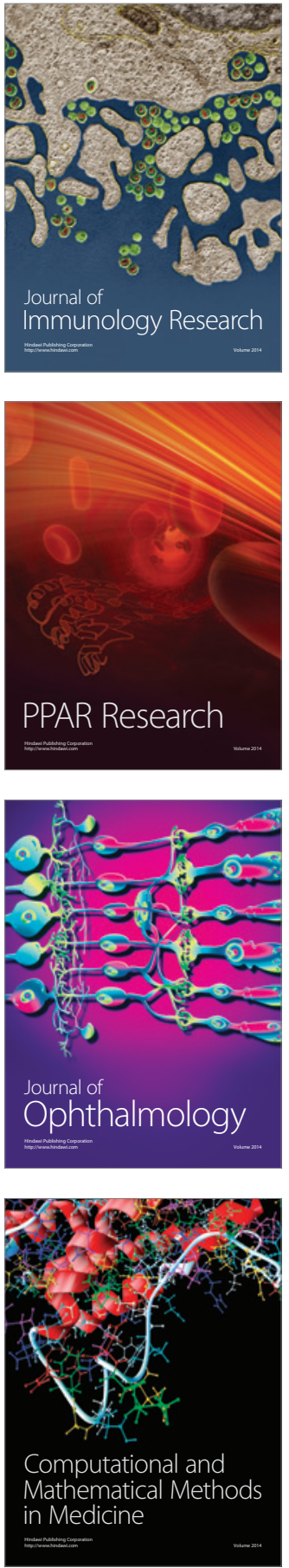

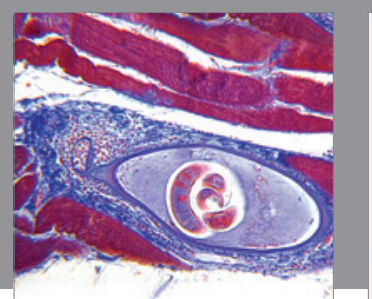

Gastroenterology Research and Practice

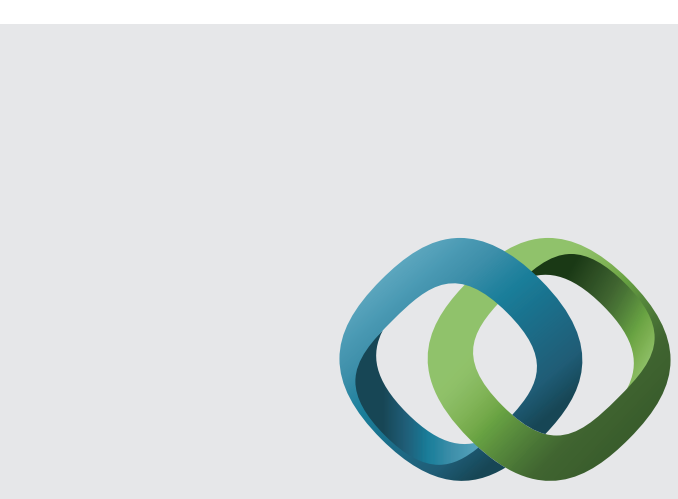

\section{Hindawi}

Submit your manuscripts at

http://www.hindawi.com
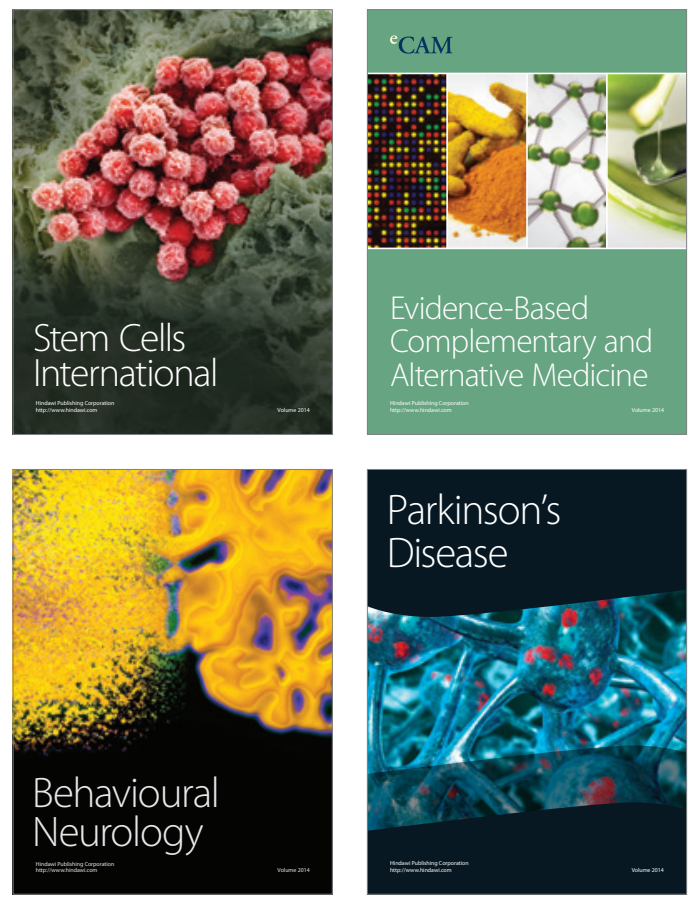
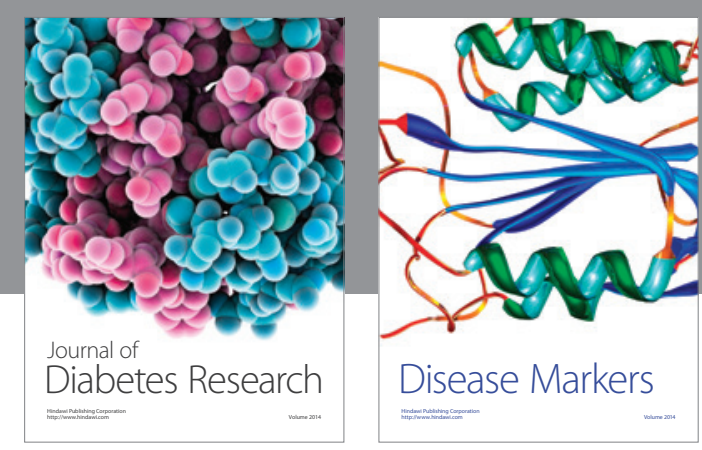

Disease Markers
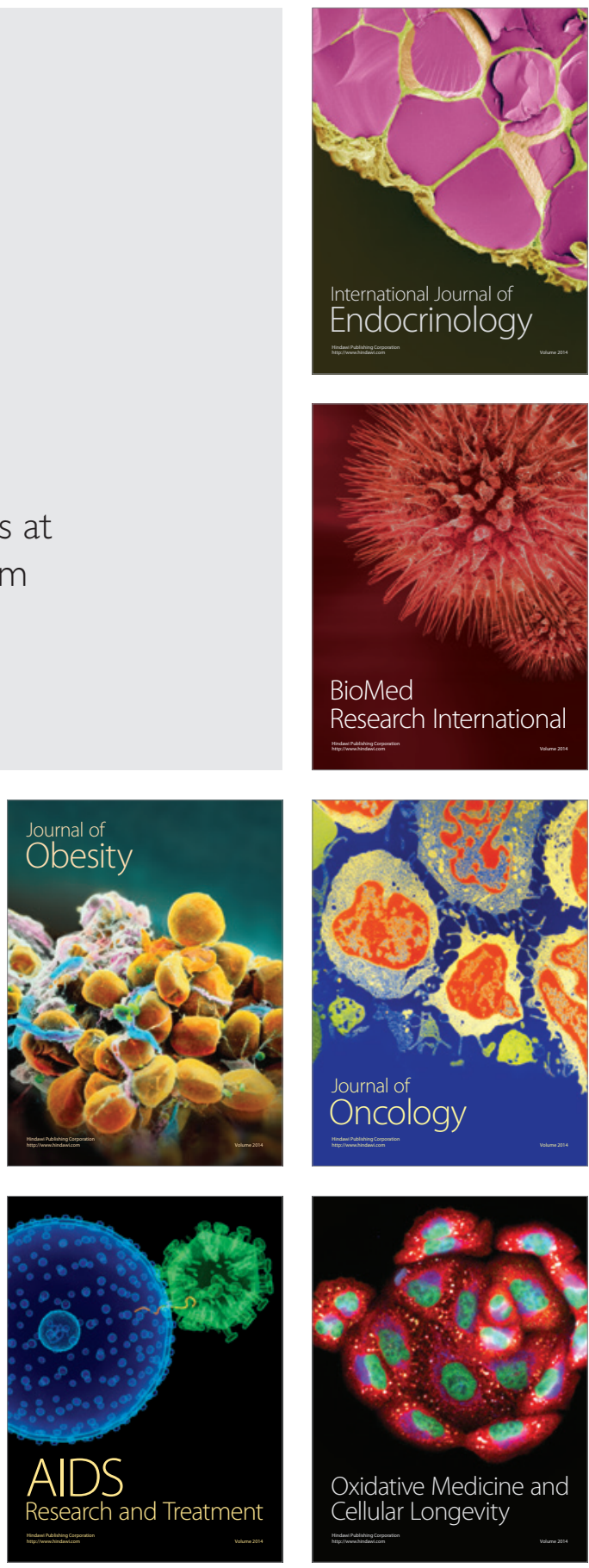